\title{
Avaliação da armadilha ovitrampa iscada com atraente natural para o monitoramento de Aedes spp. em Dili, capital do Timor-Leste
}

\author{
Evaluation of the baited ovitrap with natural attractant for \\ monitoring Aedes spp. in Dili, capital of East Timor
}

Ermelindo Barreto (in memoriam)

Marcelo Carvalho Resende (https://orcid.org/0000-0002-3731-7007) ${ }^{1}$

Alvaro Eduardo Eiras (https://orcid.org/0000-0002-3045-0673) ${ }^{1}$

Paulo Cezar Demarco Júnior (https://orcid.org/0000-0002-1593-9518) ${ }^{1}$

${ }^{1}$ Departamento de Parasitologia, Instituto de Ciências Biológicas, Universidade Federal de Minas Gerais. Av. Antônio Carlos 6627, Pampulha. 31270-010 Belo Belo Horizonte MG Brasil. marcelo.resende60@ gmail.com

\begin{abstract}
Dengue transmission has been known in East Timor since 2005, but the country is not equipped with an Aedes aegypti mosquito monitoring and control program. This study aimed to evaluate the baited ovitrap as a possible tool to monitor the arbovirus vector Dengue (DENV), Chikungunya (CHIKV) and Zika (ZIKV) and was conducted in the city of Dili, capital of EastTimor, between epidemiological weeks 32 (02/08) and 48 (02/12) of 2016. In total, 70 ovitraps were installed in residences scattered throughout fifteen streets of four Administrative Posts (districts) of the city. The following entomological indicators were used: Ovitrap Positivity Index (OPI), Vector Density Index (VDI), and Egg Density Index (EDI). A total of 158.904 eggs were collected during the experiment. The OPI showed that 98$100 \%$ of traps contained Aedes spp. in all areas of the study. The EDI and OPI indicators were positively and significantly correlated with the temperature. The two- and three-week lag for rainfall indicated a significant positive correlation for VDI and EDI. Therefore, the ovitrap is a tool that can integrate the actions of an Aedes spp. monitoring and control program in East-Timor.
\end{abstract}

Key words Aedes spp., Oviposition trap, Ovitrap, East Timor
Resumo A dengue ocorre no Timor-Leste desde 2005, porém não existe um programa de monitoramento e controle do "Aedes aegypti". O objetivo deste estudo foi de avaliar a armadilha ovitrampa iscada com atraente natural como uma possivel ferramenta para monitorar o vetor das arboviroses: Dengue (DENV), Chikungunya (CHIKV) e Zika (ZIKV). O estudo foi realizado na cidade de Dili, capital do Timor-Leste, entre as semanas epidemiológicas 32 (02/08) a 48 (02/12) de 2016. Foram instaladas 70 armadilhas Ovitrampa, em residências de 15 sucos (ruas), de quatro Postos Administrativos (bairros) da cidade. Para as analises dos dados utilizou-se os indicadores entomológicos: Indice de Positividade de Ovitrampa (IPO), Indice de Densidade Vetorial (IDV) $e$ Índice de Densidade de Ovos (IDO). Durante o experimento foram coletados 158.904 ovos de Aedes spp.. O IPO demonstrou que todas as áreas tiveram $98 \%$ a $100 \%$ de armadilhas contendo ovos de Aedes spp.. Os indicadores IDO e IPO apresentaram correlações positivas e significativa com a temperatura. A defasagem de duas e três semanas para precipitação indicou correlação positiva significativa para IDV e IDO. Portanto, a armadilha ovitrampa é uma ferramenta que pode integrar as ações de um programa de monitoramento e controle de Aedes spp. no Timor-Leste.

Palavras-chave "Aedes" spp., Armadilha de oviposição, Ovitrampa, Timor-Leste 


\section{Introdução}

Os mosquitos do gênero Aedes têm sua origem descrita no velho mundo, muito provavelmente no nordeste da África, tendo sido originalmente descrito no Egito'. São considerados mosquitos cosmopolitas, com ocorrência nas áreas tropicais e subtropicais do globo, estando sempre associado ao domicílio e peridomicílio humano ${ }^{2}$. Os espécimes de Aedes spp são considerados vetores de diversas arboviroses como a Dengue, Zika, Chikunguya e Febre Amarela ${ }^{3}$.

A compreensão da dinâmica populacional dos vetores de interesse médico em uma determinada região geográfica, por meio do monitoramento do vetor, permite o melhor planejamento de políticas públicas em saúde ${ }^{3,4}$. As estratégias para o monitoramento e controle desses insetos são necessárias para amenizar a circulação viral e garantir qualidade de vida à população $0^{5}$.

No Timor-Leste, a dengue é considerada uma importante arbovirose transmitida pelo Aedes aegypti, e os estudos são escassos principalmente sobre o monitoramento do vetor ${ }^{6}$. Diante dos consecutivos casos de dengue no Timor Leste, a partir de 2014, principalmente no município de Dili, tornou-se importante o planejamento de um programa de monitoramento do vetor, por meio de armadilhas de oviposição (ovitrampa) ${ }^{7}$.

O uso da ovitrampa é uma técnica segura, barata, rápida, eficiente e que não agride o ambien$\mathrm{te}^{8}$. Essa ferramenta permite determinar a dispersão geográfica, densidade, frequência, ocupação e sazonalidade do vetor?.

\section{Métodos}

\section{Área de estudo}

No município de Dili, capital do Timor-Leste que está localizada na costa norte da ilha do Timor-Leste e seus municípios limítrofes são Manatuto a leste, Aileu a sul, Liquiçá a oeste, e o mar de Savu a norte, que integra também a ilha de Ataúro. O município está situado na Latitude de $8^{\circ} 33^{\prime} 31^{\prime \prime}$ 'S e Longitude $125^{\circ} 34^{\prime} 25^{\prime \prime}$ E. O clima é tropical húmido com temperatura média anual variando entre $26^{\circ} \mathrm{C}$ a $32^{\circ} \mathrm{C}$ e umidade relativa do ar entre $60 \%$ a $74 \%$.

Segundo o censo demográfico do ano de 2010, o município de Dili possuía uma população de 252.884 habitantes e 39.310 domicílios, e uma área de $372 \mathrm{~km}^{2}$. O município de Dili está dividido em seis Postos Administrativos: Dom
Aleixo, Vera Cruz, Nain Feto, Cristo Rei, Metinaro e a ilha de Atauro ${ }^{10}$.

\section{Preparo da infusão natural como atraente de oviposição}

A infusão utilizada nas ovitrampas, foi preparada com 350 gramas de folhas verdes de Acácia (Leucaena leucocephala) colocada em um balde de capacidade de $15 \mathrm{~L}$, contendo $2 \mathrm{~L}$ de água. $\mathrm{O}$ balde permaneceu fechado por 48 horas para obter a fermentação das folhas.

Após este período de fermentação, a infusão foi filtrada em um pano limpo e foi utilizada a proporção de $100 \mathrm{~mL}$ de infusão e $400 \mathrm{~mL}$ de água, totalizando de $500 \mathrm{~mL}$ por dispositivo de oviposição.

\section{Substrato de oviposição (Paleta)}

Como substrato para postura de ovos, foi utilizado tecido ( $80 \%$ de algodão e $20 \%$ de poliéster, fabricado na China), de cor marrom claro e medindo $12 \mathrm{~cm}$ de comprimento por $6 \mathrm{~cm}$ de largura. O tecido apresentava um lado liso e outro rugoso, sendo que o lado rugoso ficou exposto para oviposição das fêmeas de Aedes spp.. As paletas foram colocadas verticalmente e presas por um clip no interior do dispositivo.

\section{Instalação das ovitrampas}

Foram instaladas 70 armadilhas ovitrampa em 15 sucos (ruas), de quatro Postos Administrativos (Bairro) de Dili. A seleção dos Postos Administrativos para estudo, levou em consideração a disponibilidade dos recursos humanos, sendo selecionados os Posto Administrativo de Dom Aleixo (36 ovitrampas), Cristo Rei (8 ovitrampas), Nain Feto (1 lovitrampas) e Vera Cruz (15 ovitrampas). As armadilhas foram alocadas por conveniência nos imóveis, sendo instaladas 64 dispositivos no intradomicílio (47 no quarto, 12 na cozinha, 5 no banheiro) e seis no peridomicilio do imóvel (na varanda). As instalações e vistorias dos dispositivos ficaram a cargo dos alunos da Universidade Nacional de Timor Lorosa'e.

As ovitrampas foram vistoriados regulamente a cada sete dias, para troca da paleta e infusão durante as 17 semanas de estudo. Para contagem de ovos de Aedes spp. nas paletas, utilizou a estrutura do laboratório do Departamento de Biologia da Faculdade de Educação, Artes e Humanidades da Universidade Nacional de Timor Lorosa'e. 


\section{Dados meteorológicos}

Os dados meteorológicos de temperatura média, mínima, máxima, precipitação e umidade relativa do ar do município de Dili foram fornecidos pelo Ministério de Obras Públicas do Timor-Leste.

\section{Análise dos dados}

Os ovos coletados nas ovitrampas permitiram calcular o total de ovos de Aedes spp., o Índice de Positividade da Ovitrampas (IPO= Porcentagem de armadilha com ovos de Aedes spp e as armadilhas instaladas), Índice de densidade de ovos (IDO= ovos de Aedes sp e as armadilhas positivas) e o Índice Densidade Vetorial (IDV= ovos de Aedes spp e as armadilhas vistoriadas $)^{11}$.

Os dados IPO, IDV, IDO e total de ovos de Aedes spp. foram correlacionados com os fatores abióticos (temperatura máxima, média, mínima, precipitação e umidade relativa do ar) através do teste Person. O IDV por Semana Epidemiológica foi submetido ao teste de normalidade de Shapiro-Wilks a $5 \%$ de probabilidade, apresentaram distribuição normal e, foram submetidos à Análise de Variância (ANOVA). As médias entomológicas foram comparadas a posteriori pelo teste de Tukey ao nível de significância $\mathrm{p}<0,05$.

Os dados foram processados utilizando-se os Programas: IBM SPSS versão 2.4, e os programas Excel e Word da Microsoft ${ }^{\circledR}$ Office $365^{\mathrm{TM}}{ }^{\text {. }}$.

\section{Resultados}

Durante as 17 semanas de estudo, foram coletados o total de 158.904 ovos de Aedes spp. nos quatro Postos Administrativos: Dom Aleixo, Cristo Rei, Nain Feto e Vera Cruz da capital Dili do Timor-Leste.

A maior proporção de ovos de Aedes spp. (62,5\%; 99.317 ovos) foi registrada no Dom Aleixo, seguido de Cristo Rei (16\%; 25.396 ovos), Nain Feto (12\%; 19.056 ovos) e o Vera Cruz apresentou a menor proporção (9,2\%; 14.585 ovos).

A média de ovos de Aedes spp. não apresentou diferença significativa por ambiente de instalação das ovitrampas nas residências de Dili (Anova $\mathrm{F}_{(4}$ $\left.{ }_{289)}=0,678 ; \mathrm{p}>0,05\right)$ onde no ambiente intradomicíliar representados pelo quarto coletou em média: 129 ovos, na cozinha 141 ovos, no banheiro 146 ovos, e no ambiente peridomicíliar representado pela varanda 134 ovos de Aedes spp..
Através dos indicadores fornecidos pelas ovitrampas, observou que o IDV e IDO apresentaram comportamento semelhante nos quatro Postos Administrativos avaliados, onde o Dom Aleixo variou entre 109,6 a 199,3; Cristo Rei entre 93 a 134,4; Nain Feto variou entre 73,4 a 143,4; e Vera Cruz variou entre 85,5 a 129,4. Ressalta-se o Posto Administrativo Dom Aleixo que apresentou IDO acima de 100 ovos de Aedes spp. durante todo o período experimental e atingindo um pico de 199 ovos na Semana Epidemiológica 43 (Tabela 1).

O IDV no município não observou diferença significativa durante o período experimental (Anova, $\mathrm{F}_{(17,68)}=1,03 ; \mathrm{p}>0,05$ ) (Figura 1).

O IPO nos Postos Administrativos Dom Aleixo, Nain Feto e Vera Cruz, variaram entre 98,03 a $100 \%$, e o Posto Administrativo Cristo Rei apresentou durante todo o período experimental $100 \%$ das armadilhas positivas com ovos de Aedes spp..

Durante o estudo, as temperaturas médias, mínimas e máximas variaram entre 26 e $32^{\circ} \mathrm{C}$, 16 e $24^{\circ} \mathrm{C}$ e 28 e $35^{\circ} \mathrm{C}$, respectivamente. Grandes oscilações foram observadas para a precipitação semanal, entre os meses de agosto a setembro (SE 32 a 39) de 2016, ocorreu uma precipitação média de $0,21 \mathrm{~mm}$, enquanto durante as semanas epidemiológicas restantes, uma média de $1,53 \mathrm{~mm}$ de precipitação representando o período chuvoso de outubro a dezembro (Figura 2). A umidade relativa semanal média variou entre $58 \%$ e $72 \%$.

A análise de correlação de Pearson, indicou uma relação positiva e significativa entre total de ovos, IDV e IDO com as variáveis de temperatura máxima, média, e mínima, para o período de estudo. Para as variáveis entomológicas fornecidas pela ovitrampa e correlacionada com a umidade relativa do ar e precipitação na mesma semana não foi observada uma correlação significativa. Observa-se que umidade relativa apresentou correlação negativa para IDV, IPO e IDO, e a precipitação para total de ovos e IPO (Tabela 2).

No entanto, os indicadores entomológicos fornecidos pela ovitrampa quando correlacionados com a precipitação pluviométrica, sob o efeito da defasagem de uma, duas, três e quatro semanas, observou que a ação de protelar em duas semanas $\left(r^{2}=0,518, p=0,0332\right)$ e três semanas $\left(r^{2}=0,5034, p=0,0394\right)$ apresentou correlação positiva significativa para as variáveis IDV e IDO (Tabela 3). 
Tabela 1. Variáveis entomológicas fornecidas pela armadilha ovitrampa na cidade de Dili, Timor-Leste, no período de agosto a dezembro de 2016 (SE 32 a 48).

\begin{tabular}{|c|c|c|c|c|c|c|c|c|c|c|c|c|}
\hline \multirow{3}{*}{$\begin{array}{c}\text { Semana } \\
\text { Epidemiológica }\end{array}$} & \multicolumn{12}{|c|}{ Variáveis Entomológicas } \\
\hline & \multicolumn{3}{|c|}{ Dom Aleixo } & \multicolumn{3}{|c|}{ Cristo Rei } & \multicolumn{3}{|c|}{ Nain Feto } & \multicolumn{3}{|c|}{ Vera Cruz } \\
\hline & IDV & IDO & IPO & IDV & IDO & IPO & IDV & IDO & IPO & IDV & IDO & IPO \\
\hline 32 & 116,5 & 116,5 & 100 & 94,9 & 94,9 & 100 & 84,9 & 84,9 & 81,8 & 95,9 & 95,9 & 100 \\
\hline 33 & 133,9 & 133,9 & 100 & 93 & 93 & 100 & 106,6 & 106,6 & 100 & 93,7 & 93,7 & 100 \\
\hline 34 & 123,3 & 123,3 & 100 & 105,8 & 105,8 & 100 & 83,6 & 83,6 & 100 & 86,7 & 86,7 & 93,3 \\
\hline 35 & 129,5 & 129,5 & 97,2 & 95,3 & 95,3 & 100 & 81,5 & 81,5 & 100 & 95,7 & 95,7 & 93,3 \\
\hline 36 & 109,6 & 109,6 & 100 & 93,1 & 93,1 & 100 & 82,5 & 82,5 & 100 & 93,5 & 93,5 & 93,3 \\
\hline 37 & 119,8 & 119,8 & 100 & 98,6 & 98,6 & 100 & 82,1 & 82,1 & 100 & 93,5 & 93,5 & 100 \\
\hline 38 & 141,2 & 141,2 & 100 & 95,3 & 95,3 & 100 & 73,4 & 73,4 & 100 & 94,6 & 94,6 & 100 \\
\hline 39 & 153,7 & 153,7 & 100 & 106,9 & 106,9 & 100 & 94,4 & 94,4 & 100 & 96,1 & 96,1 & 100 \\
\hline 40 & 191,3 & 191,3 & 100 & 109,4 & 109,4 & 100 & 106,2 & 106,2 & 100 & 85,5 & 85,5 & 100 \\
\hline 41 & 191,7 & 191,7 & 100 & 101,5 & 101,5 & 100 & 97,4 & 97,4 & 100 & 112,1 & 112,1 & 100 \\
\hline 42 & 194,7 & 194,7 & 100 & 99,6 & 99,6 & 100 & 109,2 & 109,2 & 90,9 & 105 & 105 & 100 \\
\hline 43 & 199,3 & 199,3 & 100 & 108,8 & 108,8 & 100 & 100,2 & 100,2 & 100 & 108,4 & 108,4 & 100 \\
\hline 44 & 196,3 & 196,3 & 100 & 126,1 & 126,1 & 100 & 99,7 & 99,7 & 100 & 105,5 & 105,5 & 100 \\
\hline 45 & 190,4 & 190,4 & 100 & 122,9 & 122,9 & 100 & 115,4 & 115,4 & 100 & 105,3 & 105,3 & 100 \\
\hline 46 & 194 & 194 & 100 & 118,6 & 118,6 & 100 & 133,3 & 133,3 & 100 & 97,5 & 97,5 & 86,7 \\
\hline 47 & 191 & 191 & 100 & 120,4 & 120,4 & 100 & 138,5 & 138,5 & 100 & 94,8 & 94,8 & 100 \\
\hline 48 & 182,7 & 182,7 & 100 & 134,4 & 134,4 & 100 & 143,4 & 143,4 & 100 & 129,4 & 129,4 & 100 \\
\hline
\end{tabular}

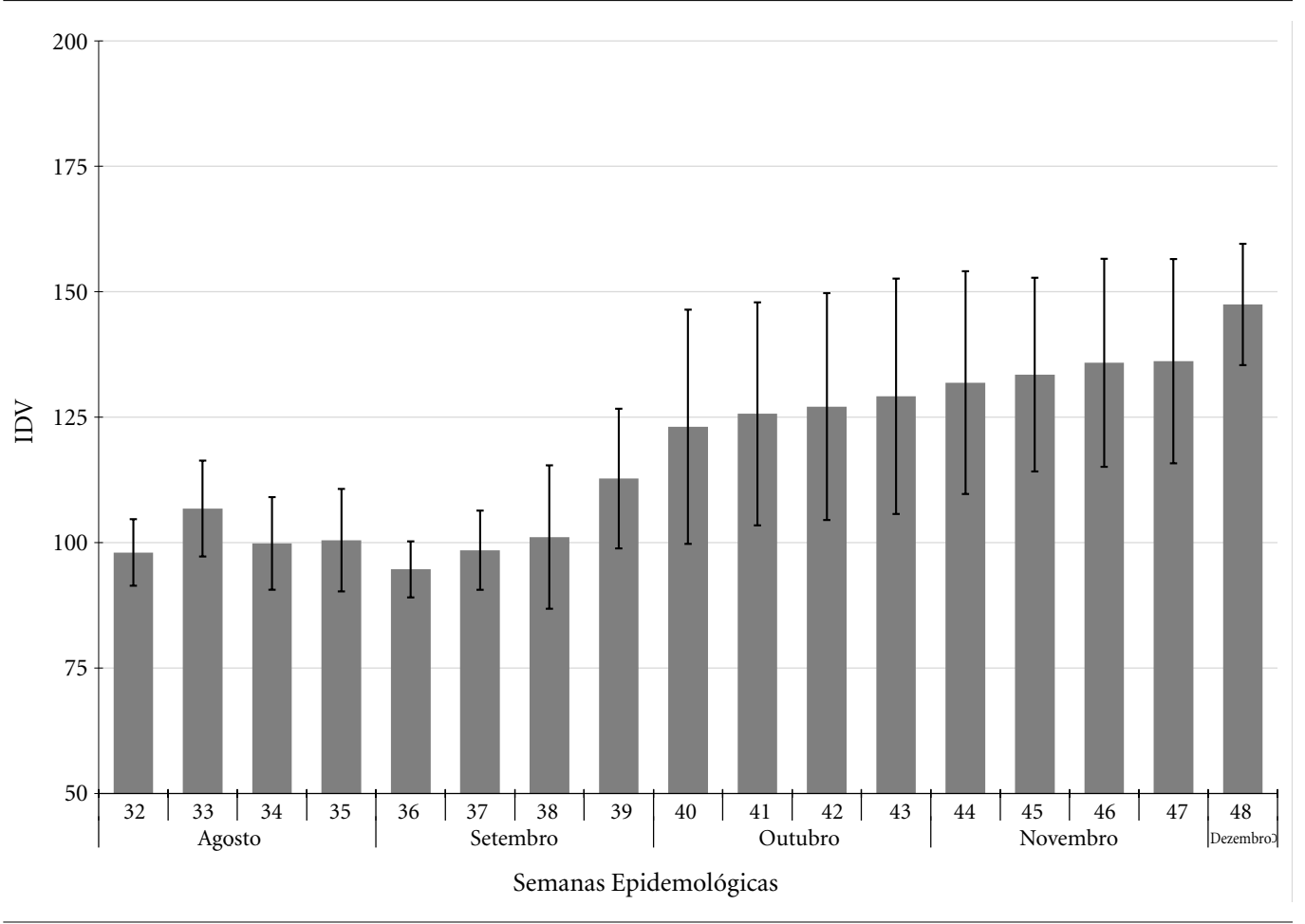

Figura 1. Índice de Densidade Vetorial ( $\mu \pm$ erro padrão) de Aedes spp. por semana epidemiológica na cidade de Dili, capital do Timor-Leste, no período de agosto a dezembro de 2016. 


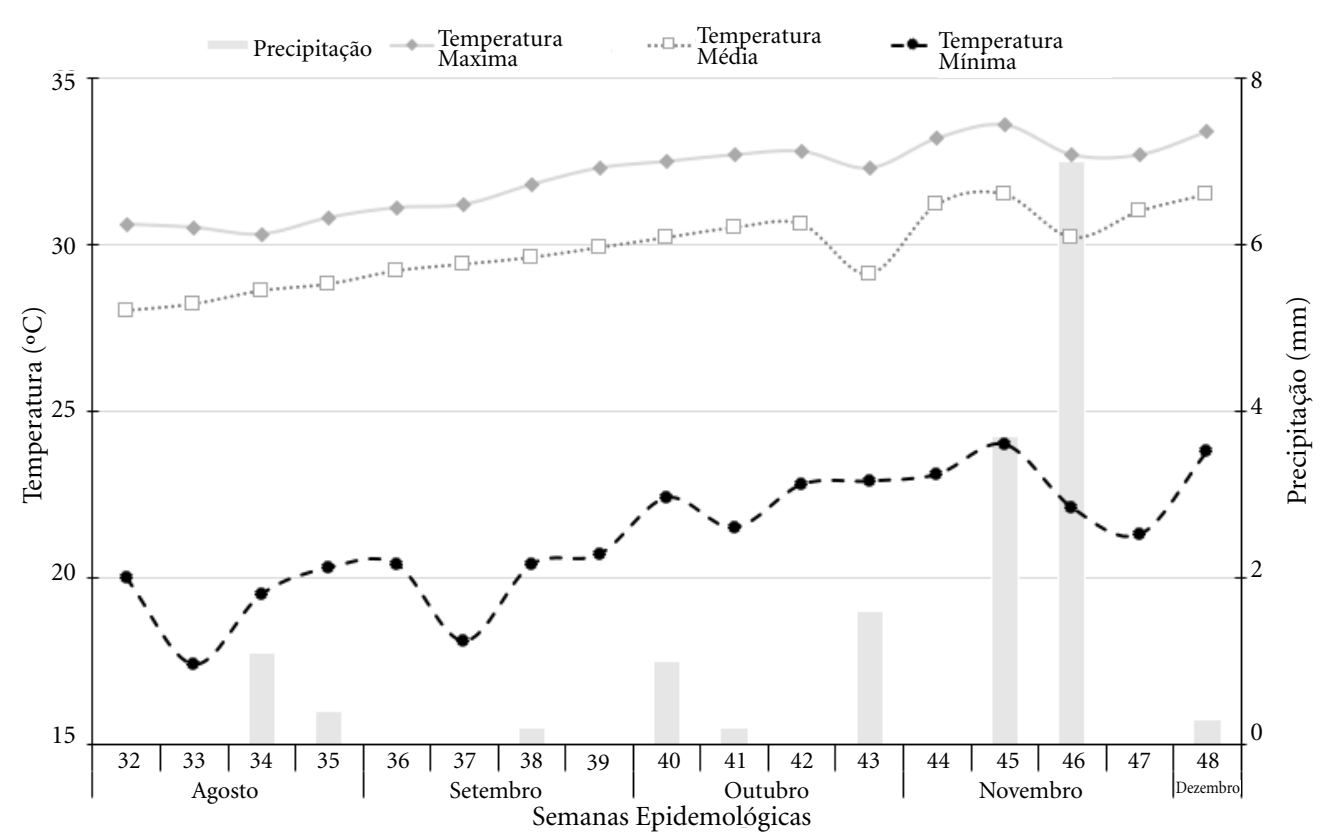

Figura 2. Variáveis meteorológicas semanais na cidade de Dili, capital do Timor-Leste, no período de agosto a dezembro de 2016.

Tabela 2. Correlação Pearson entre as variáveis abióticas em relação total de ovos coletados, IDV, IPO e IDO na cidade de Dili, Timor-Leste, no período de agosto a dezembro de 2016.

\begin{tabular}{|c|c|c|c|c|c|}
\hline \multirow{3}{*}{\multicolumn{2}{|c|}{$\begin{array}{l}\text { Variáveis } \\
\text { Abióticas }\end{array}$}} & \multicolumn{4}{|c|}{ Variáveis Entomológicas } \\
\hline & & \multirow{2}{*}{$\begin{array}{c}\text { Total de ovos } \\
\text { Coef.Corr. (p) } \\
\end{array}$} & \multirow{2}{*}{$\begin{array}{c}\text { IDV } \\
\text { Coef.Corr. (p) }\end{array}$} & \multirow{2}{*}{$\begin{array}{c}\text { IPO } \\
\text { Coef.Corr. (p) } \\
\end{array}$} & \multirow{2}{*}{$\begin{array}{c}\text { IDO } \\
\text { Coef.Corr. (p) }\end{array}$} \\
\hline & & & & & \\
\hline \multirow[t]{3}{*}{$\mathrm{T}$} & Máx. & $0,907(<0,0001)^{\star}$ & $0,8736(<0,0001)^{*}$ & $0,1876(0,4708)$ & $0,8736(<0,0001)^{\star}$ \\
\hline & Méd. & $0,805(<0,0001)^{*}$ & $0,7954 \cdot(0,0001)^{\star}$ & $0,176(0,4993)$ & $0,7954 \cdot(0,0001)^{\star}$ \\
\hline & Mín. & $0,827(<0,0001)^{\star}$ & $0,7879(0,0002)^{\star}$ & $0,2178(0,4011)$ & $0,7879(0,0002)^{\star}$ \\
\hline UR & Méd. & $0,369(0,1444)$ & (-) 0,2186 (0,3993) & $(-) 0,3177(0,2140)$ & $(-) 0,2186(0,3993)$ \\
\hline $\mathrm{PP}$ & Méd. & $(-) 0,2208(0,3945)$ & $0,4775(0,0526)$ & $(-) 0,1303(0,6181)$ & $0,4775(0,0526)$ \\
\hline
\end{tabular}

Abreviaturas: $\mathrm{T}=$ Temperatura; Máx. $=$ Máxima; Méd. = Média; Mín. $=$ Mínima; $\mathrm{UR}=$ Umidade relativa do ar; $\mathrm{PP}=$ Precipitação pluviométrica. ${ }^{*}$ Correlação significativa entre as variáveis

Tabela 3. Correlação Pearson entre a variável meteorológica precipitação em relação total de ovos coletados, IDV, IPO e IDO na cidade de Dili, Timor-Leste, no período de agosto a dezembro de 2016.

\begin{tabular}{lcrrr}
\hline \multirow{2}{*}{ Defasagem Semanal } & \multicolumn{4}{c}{ Variáveis Entomológicas } \\
\cline { 2 - 5 } & Total de ovos & \multicolumn{1}{c}{ IDV } & \multicolumn{1}{c}{ IPO } & \multicolumn{1}{c}{ IDO } \\
\cline { 2 - 5 } & Coef.Corr. $(\mathbf{p})$ & \multicolumn{1}{c}{ Coef.Corr. $(\mathbf{p})$} & \multicolumn{1}{c}{ Coef.Corr. $(\mathbf{p})$} & \multicolumn{1}{c}{ Coef.Corr. $(\mathbf{p})$} \\
\hline 1 semana & $0,3668(0,1476)$ & $0,4321(0,0832)$ & $(-) 0,0977(0,7091)$ & $0,4321(0,0832)$ \\
2 semana & $0,4113(0,1010)$ & $0,518(0,0332)^{\star}$ & $0,01647(0,9500)$ & $0,518(0,0332)^{\star}$ \\
4 semana & $0,3606(0,1550)$ & $0,5034(0,0394)^{*}$ & $(-) 0,129(0,6218)$ & $0,5034(0,0394)^{\star}$ \\
\hline
\end{tabular}

${ }^{*}$ Correlação significativa entre as variáveis. 


\section{Discussão}

A armadilha de oviposição vem sendo empregada em diversos países para o monitoramento de Aedes spp. ${ }^{8,9,11}$. No município de Dili a ovitrampa revelou-se uma ferramenta com alta sensibilidade para o acompanhamento da infestação do gênero Aedes, proporcionando a obtenção de dados em intervalos curtos (semanais) e de forma continua.

O monitoramento semanal, destacou o Posto Administrativo Dom Aleixo com 62,5\% dos ovos coletados em relação aos Postos Administrativos Cristo Rei, Nain Feto e Vera Cruz. O elevado percentual de ovos coletados no Posto Administrativo de Dom Aleixo pode ser atribuído por ser uma área mais urbanizada da cidade de Dili e apresentar crescimento desornado com um sistema de abastecimento de água irregular, provavelmente contribuíram para a maior prevalência de ovos de Aedes spp. nesta área ${ }^{12}$.

Ao analisarmos os ambientes para instalação das armadilhas de oviposição, não houve constatação de diferenças significativas nas médias de ovos de Aedes spp. coletados nas ovitrampas, instaladas no intradomicílio (quarto, cozinha e banheiro) ou no peridomicílio (varanda). Apesar de não observar uma diferença entre os ambientes analisados, ressalta-se a proporção elevada de fêmeas de Aedes spp. que fazem postura em criadouros dentro dos imóveis. O fenómeno deve-se a más condições das habitações em Dili, moradias construídas com aberturas nas paredes permitindo a livre circulação dos mosquitos do gênero Aedes ${ }^{7}$. Estes resultados diferem dos achados no Brasil, onde observa-se que as maiorias dos criadouros de Aedes spp. encontram-se no peridomicílio $^{12,13}$.

O IDV semanal de Aedes spp., fornecidos pela armadilha ovitrampa em Dili (Timor-Leste) não apontou diferenças significativas, com maior e menor infestação, durante o período experimental.

O IPO da ovitrampa permitiu avaliar qualitativamente a presença de Aedes spp., demonstrando que os Postos Administrativos Dom Aleixo, Cristo Rei, Naim Feto e Vera cruz foram similares e variou entre 98 a 100\%. O Posto Administrativo Cristo Rei apesar de não ter os maiores IDO e IDV, suas armadilhas ficaram positivas durante todo o experimento. Esse fenômeno de positividade pode estar associado ao comportamento da fêmea de Aedes spp. que não deposita todos os seus ovos em um único criadouro, distribuindo-o em diferentes recipientes ${ }^{13}$. Este comporta- mento denominado "oviposição aos saltos" (skip oviposition) permitiu a mesma fêmea depositar os ovos em mais de uma armadilha ${ }^{14}$.

Os dados de IPO nos Postos administrativos da cidade de Dili, permitiu indicar que as áreas estudadas são de alto risco para transmissão de dengue e outras arboviroses por Ae. aegypti e Ae. albopictus. Segundo a literatura, o indicador IPO igual ou maior que $40 \%$ de positividade indica risco de transmissão de arboviroeses ${ }^{11}$.

O IDO e IDV de Aedes spp. por Posto Administrativo de Dili, variaram de forma semelhante, e na maioria das semanas epidemiológicas foi superior a 100 ovos de Aedes spp. coletados por ovitrampa positiva e inspecionada, respectivamente. Este comportamento dos indicadores IDO e IDV, são atribuídos ao alto índice de infestação populacional de Aedes spp. ${ }^{13,14}$, encontrados nos Postos administrativos avaliados.

Os altos índices fornecidos pelas armadilhas de oviposição na cidade de Dili, pode estar associado ao crescimento populacional desordenado como consequência da guerra civil que trouxe desequilíbrios na urbanização ${ }^{10}$. Observa-se que o abastecimento de água na cidade é irregular e a ausência de uma rede coletora de esgoto, onde os resíduos cloacais das residências permanecem em céu aberto, características de ocupação da área urbana que estimulam a proliferação e dispersão do Aedes spp. e outros culicídeos ${ }^{12,13}$.

Em relação as variáveis abióticas e os índices de infestação de Aedes spp. em Dili, verificou-se que houve uma correlação significativa entre os indicadores IDV, IDO e o número total de ovos de Aedes spp coletados com a temperatura. Não foi observado associação positiva da precipitação na mesma semana epidemiológica e umidade relativa do ar. A precipitação foi correlacionada positivamente com os indicadores IDV e IDO somente quando os dados foram defasados com duas e três semanas. Provavelmente esta desfasagem de tempo permitiu ajustar a variável precipitação em função do ciclo biológico do $A e$. Aegypti e Ae albopictus. Esse tempo de duas e três semanas está relacionada com aparecimento de criadouros potências, após o período chuvoso ${ }^{14}$.

Os dados de correlação positiva com a temperatura que atua como um fator modelador do processo de infestação por Aedes spp estão de acordo com os resultados da literatura especifica $^{15,16}$, que enfatizou a importância da temperatura sobre a distribuição do Aedes spp.. No Timor-Leste as estações climáticas podem ser divididas em dois períodos, o seco que ocorre entre os meses de maio a outubro e o período chuvoso 
que ocorre entre os meses de novembro a abril.

As condições climáticas são fatores determinantes da existência e manutenção de Ae. aegypti no meio ambiente ${ }^{16}$. Desta maneira, a temperatura ideal para o mosquito Ae. aegypti para sua proliferação seria entre $24^{\circ} \mathrm{C}$ e $28^{\circ} \mathrm{C}$ e a umidade relativa do ar ideal acima de $70 \%$. Nessas condições o Ae. aegypti se reproduz com maior intensidade, aumenta a sua sobrevida, ovipostura, atividade hematofágica e a eficiência da reprodução do vírus em seu interior ${ }^{15}$. Verifica-se que estes padrões meteorológicos ocorreram durante todo o período experimental na cidade de Dili.
Portanto, o presente estudo sugere a utilização de armadilha de oviposição (ovitrampa) em Dili, Timor-Leste, como um método apropriado de detecção da presença e a variação da densidade populacional de Ae. aegypti em diferentes ambientes. Portanto, o Ministério da Saúde do Timor-Leste poderá utilizar os indicadores fornecidos pela armadilha de oviposição para direcionar as atividades de controle do Aedes spp., permitindo de forma mais eficaz atuar nas áreas de abrangência das armadilhas positivas e nas áreas com valores de IDO e IDV que indiquem risco de transmissão de arboviroses por Aedes spp..

\section{Colaboradores}

E Barreto: Organização dos testes de campo, treinamento e orientação dos alunos de iniciação cientifica que participaram do projeto, tabulação dos resultados prévios. MC Resende: Elaboração dos protocolos dos testes de campo, acompanhamento dos testes de campo, elaboração das discussões do estudo e tabulação dos resultados finais. AE Eiras: Coordenador da pesquisa, trabalhou na elaboração dos protocolos de campo, acompanhamento dos testes de campo, revisor final. PC Demarco Júnior: Elaboração e padronização do material escrito, elaboração dos testes estatístico e resultados. 


\section{Referências}

1. Eiras AE. Família Culicidae. In: Neves DP, Melo AL, Genaro O, Linardi PM, organizadores. Parasitologia Humana. São Paulo: Atheneu; 2015. p. 434-437.

2. Forattini OP. Culicidologia Médica: identificação, biologia e epidemiologia. 2a ed. São Paulo: Edusp; 2002.

3. Consoli RAGB, Oliveira RL. Principais mosquitos de importância no Brasil. Rio de Janeiro: Fiocruz; 1994.

4. Brasil. Ministério da Saúde (MS), FUNASA. Programa Nacional de Controle da Dengue. Brasília: MS; 2002.

5. Figueiredo MLG, Gomes AC, Amarilla AA, Leandro AS, Orrico AS, Araujo RF, SM Castro J, Durigon EL, Aquino VH, Figueiredo LT. Mosquitoes infected with dengue viruses in Brazil. Virol J 2010; 12(7):152-157.

6. World Health Organization (WHO). Dengue Guidelines for Diagnosis, Treatment, Prevention and Control. New Edition. Genebra: WHO; 2009.

7. Whelan P, Hapgoog GA. Mosquito Survey of Dili, East Timor, and Implications for Disease Control Department of Health and Community Services. Med. Entomol. 2000; 1(12):405-416.

8. Beserra EB, Ribeiro OS, Oliveira SA. Flutuação populacional e comparação de métodos de coleta de Aedes (Stegomyia) aegypti (Diptera, Culicidae). Iheringia, Sér. Zool. 2014; 5(4):418-425.

9. Roque RA. Avaliação de armadilhas iscadas com infusões de gramíneas como atraentes e/ou estimulantes de oviposição do mosquito Aedes (Stegomyia) sp. (Diptera: Culicidae) [dissertação]. Belo Horizonte: Universidade Federal Minas Gerais; 2002.

10. Deen J, Matos LC, Temple B, Su JY, da Silva J, Liberato S, Silva V, Soares AI, Joshi V, Moon S, Tulloch J, Martins J, Mulholland K. Identifying national health research priorities in Timor-Leste through a scoping review of existing health data. Health Res Policy Syst 2013; 11(1):8-10.
11. Gomes ADC. Medidas dos níveis de infestação urbana para Aedes (Stegomyia) aegypti e Aedes (Stegomyia) albopictus em programa de vigilância entomológica. Info. Epid. do SUS 1998; 17(7):49-57.

12. Forattini OP, Brito M. Reservatórios domiciliares de água e controle do Aedes aegypti. Rev Saude Publica 2003; 29 (5):676-677.

13. Domingos MDF. Aspectos da ecologia de Aedes aegypti (Linnaeus) em Santos, São Paulo, Brasil [tese]. São Paulo: Escola de Saúde Pública; 2005.

14. Micieli MV, Campos RE. Oviposition activity and seasonal pattern of a population of Aedes (Stegomyia) aegypti (L.)(Diptera: Culicidae) in subtropical Argentina. Mem Inst Oswaldo Cruz 2003; 18(7):659-663.

15. Ajuz LC, Vestena LR. Influence of Rainfall and Temperature Ambient on Longevity and Fertility of Aedes aegypti and Aedes albopictus in the City of Guarapuava-PR and the Possibility of Superinfestation. Revista Brasileira de Geografia Médica e da Saúde 2013; 10(7):1-18.

16. Calado DC, Navarro-Silva MA. Exigências térmicas de Aedes (Stegomyia) albopictus Skuse, 1894 (Diptera, Culicidae) em condições de laboratório. Rev. Bras. Entomol.. 2002; 27(7):547-555.

Artigo apresentado em 02/03/2018

Aprovado em 01/06/2018

Versão final apresentada em 03/06/2018 